\title{
A switching mechanism in periodically driven quantum systems with dissipation
}

\author{
Roland Ketzmerick and Waltraut Wustmann \\ Institut für Theoretische Physik, Technische Universität Dresden, 01062 Dresden, Germany
}

(Dated: November 3, 2018)

\begin{abstract}
We introduce a switching mechanism in the asymptotic occupations of quantum states induced by the combined effects of a periodic driving and a weak coupling to a heat bath. It exploits one of the ubiquitous avoided crossings in driven systems and works even if both involved Floquet states have small occupations. It is independent of the initial state and the duration of the driving. As a specific example of this general switching mechanism we show how an asymmetric double well potential can be switched between the lower and the upper well by a periodic driving that is much weaker than the asymmetry.
\end{abstract}

PACS numbers: 05.30.-d, 05.70.Ln, 05.45.Mt

\section{INTRODUCTION}

The interplay between a coherent periodic driving force and the incoherent damping of a thermal environment enriches the dynamics of a quantum system and opens new potential applications [1]. In addition to controlling the transient dynamics, e.g. with respect to tunneling [2, 3, 4], a control of the asymptotic state is desirable. The ability to design a system's probability distribution, e.g. to switch between two macroscopically distinguishable states, in the presence of a thermal environment is a key to quantum control techniques.

The paradigmatic model for switching is a double well potential which is experimentally realized in superconducting quantum interference devices (SQUIDs) [5], atom-optical potentials [6, 7], spin tunneling in condensed matter 8] or in the transfer of protons along chemical bonds [9]. In some cases the model can be restricted to a two-level system. Different approaches for switching by a population inversion in driven twolevel systems have been proposed, e.g. induced by symmetry-breaking [10], structured environments 11] strong nonequilibrium noise [12], or strong driving [13]. However, the restriction to a two- or a three-level system limits the possible switching mechanisms.

Time-periodic quantum systems are best described by Floquet states, which are solutions of the Schrödinger equation without the coupling to the environment. When coupling the time-periodic system weakly to a thermal bath, all Floquet states are asymptotically populated with occupation probabilities, which can be determined within a Floquet-Markov approach [14, 15, 16, 17, 18]. These occupations are quite different from the canonical distribution of Boltzmann weights in undriven systems and so far lack an intuitive understanding.

In this paper we demonstrate a dramatic property of time-periodically driven quantum systems weakly coupled to the environment: The asymptotic state can be switched to an almost orthogonal state by a small parameter variation. This is in stark contrast to timeindependent systems, where the asymptotic occupations are determined by Boltzmann weights and vary slowly with a parameter. The proposed switching mechanism exploits one of the ubiquitous avoided crossings in driven systems and works even if both involved Floquet states have small occupations. As a specific example of this general switching mechanism we show for an asymmetric double well potential, see Fig. 1 that a weak periodic driving switches the cycle-averaged asymptotic probability density from the ground state of the undriven system in the left well to the right well. Note, that the periodic driving is much weaker than the asymmetry, see Fig. 1(a), and therefore this switching is unrelated to previous studies on hysteretic switching in a driven dissipative double well [19, 20]. We explain the switching mechanism by an effective rate equation, which combines the effects of the

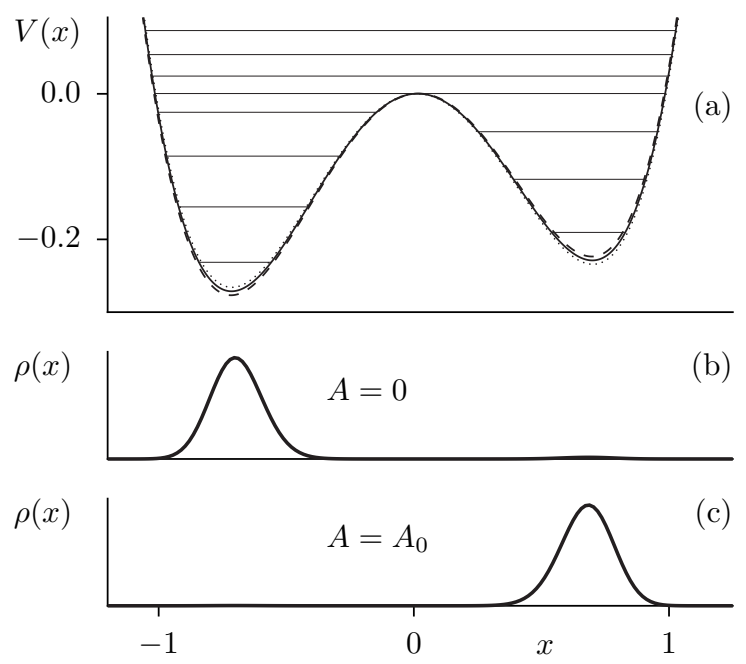

FIG. 1: a) Asymmetric double well potential and its eigenenergies without driving, $A=0$ (solid line), and the almost indistinguishable variation of the potential for a small driving amplitude $A_{0} \approx 0.008$ (dashed and dotted line). b) Asymptotic probability density $\rho(x)$ for $A=0$ and a small temperature $1 / \beta=1 / 100$, with almost all probability in the left well. c) Cycle-averaged asymptotic probability density $\rho(x)$ according to Eq. 5 for $A=A_{0}$, with more than $99 \%$ of probability in the right well, demonstrating a weak driving induced switching to a macroscopically different state. See Fig. 2 for parameters. 
coherent driving at an avoided crossing of two Floquet states with the incoherent bath coupling.

The paper is organized as follows: In Sec. II our model for the periodically driven, dissipative double well is introduced. The switching mechanism is investigated in Sec. III. We finally conclude and discuss advantages of the switching mechanism in Sec. IV]

\section{THE MODEL SYSTEM}

As an example we study a particle in an asymmetric double well potential in the quantum regime, where the ground state is in the left well and the first excited state is in the right well, see Fig. 1(a). It is driven by an additive time-periodic force, leading to the system Hamiltonian

$$
H_{s}(t)=\frac{p^{2}}{2 m}+V_{0}\left[\frac{x^{4}}{x_{0}^{4}}-\frac{x^{2}}{x_{0}^{2}}+\frac{x}{x_{0}}(\mu+A \cos \Omega t)\right],
$$

where $\mu$ is the asymmetry parameter of the double well potential and $A$ and $\Omega$ are the driving amplitude and frequency, respectively. We introduce the dimensionless quantities $\tilde{x}=x / x_{0}, \tilde{H}_{s}=H_{s} / V_{0}, \tilde{t}=t \cdot V_{0} / \hbar, \tilde{\Omega}=$ $\Omega \cdot \hbar / V_{0}$, and $\hbar_{\mathrm{eff}}=\hbar /\left(\sqrt{m V_{0}} x_{0}\right)$. In the following we omit the tilde and then the dimensionless Hamiltonian reads

$$
H_{s}(t)=-\frac{\hbar_{\mathrm{eff}}^{2}}{2} \frac{\partial^{2}}{\partial x^{2}}+x^{4}-x^{2}+x(\mu+A \cos \Omega t) .
$$

The Schrödinger equation of a periodically driven quantum system has according to the Floquet theorem solutions of the form $\psi_{i}(t)=e^{-\mathrm{i} \varepsilon_{i} t} u_{i}(t)$, with $u_{i}(t+T)=$ $u_{i}(t)$ and $T=2 \pi / \Omega$ the period of the driving. The timeperiodic parts $u_{i}(t)$ of the Floquet states form a complete orthonormal set at all times. The quasienergies $\varepsilon_{i}$ can be chosen to lie in the interval $[0, \Omega)$.

The coupling to a heat bath is modeled in a standard way by a Hamiltonian [21]

$$
H(t)=H_{s}(t)+H_{b}+H_{s b} .
$$

The bath Hamiltonian $H_{b}=\sum_{n}\left(\frac{p_{n}^{2}}{2 m_{n}}+\frac{m_{n} \omega_{n}^{2}}{2} x_{n}^{2}\right)$ describes an ensemble of noninteracting harmonic oscillators coupled via $H_{s b}=x \sum_{n} c_{n} x_{n}$ to the system. The properties of the system-bath coupling are given in terms of the spectral density of the bath $J(\omega):=$ $\frac{\pi}{2} \sum_{n} \frac{c_{n}^{2}}{m_{n} \omega_{n}}\left[\delta\left(\omega-\omega_{n}\right)-\delta\left(\omega+\omega_{n}\right)\right]$. In the continuum limit the spectral density is assumed to be a smooth function which is linear for an Ohmic bath. An exponential cutoff beyond the spectral mode $\omega_{c}$ leads to $J(\omega)=\eta \omega e^{-|\omega| / \omega_{c}}$, where $\eta$ is proportional to the classical damping coefficient.

In the presence of the heat bath the state of the system is described by the reduced density operator $\rho(t)$. Its equation of motion for time-periodic quantum systems has been derived within the Floquet-Markov approach 14, 15, 16, 17, 18]: Herein the Floquet formalism ensures a non-perturbative treatment of the driven systems coherent dynamics. The coupling to the heat bath is treated perturbatively, which is valid in the limit of weak coupling between the driven system and the bath. This approximation requires a rapid decay of bath correlations compared to the typical relaxation time of the system and we further require $\Omega \ll \omega_{c}$. In the following we restrict the discussion to the limit of large times, larger than the relaxation time. In this limit the density matrix $\rho_{i j}$ in the basis of the periodic parts $u_{i}(t)$ of the Floquet states is approximated as timeindependent [15, 18]. Note, that the corresponding density operator, $\sum_{i, j}\left|u_{i}(t)\right\rangle \rho_{i j}\left\langle u_{j}(t)\right|$, is time-periodic because of the inherent time-dependence of the $u_{i}(t)$. The matrix elements $\rho_{i j}$ obey the rate equation

$$
\begin{aligned}
& \mathrm{i}\left(\varepsilon_{i}-\varepsilon_{j}\right) \rho_{i j}= \\
& \quad-\sum_{k, l}\left\{\rho_{l j} R_{i k ; l k}+\rho_{i l} R_{j k ; l k}^{*}-\rho_{k l}\left(R_{l j ; k i}+R_{k i ; l j}^{*}\right)\right\} .
\end{aligned}
$$

The complex rates $R_{i j ; k l}=\pi \sum_{m} x_{i j}(m) x_{k l}^{*}(m) g\left(\varepsilon_{l}-\right.$ $\left.\varepsilon_{k}-m \Omega\right)$ describe bath-induced transitions between the Floquet states, the $x_{i j}(m)$ are the Fourier coefficients of the time-periodic matrix elements $\left\langle u_{i}(t)|x| u_{j}(t)\right\rangle$, and $g(\omega)$ is the correlation function of the bath coupling operator. The latter is given by $g(\omega)=n_{\beta}(\omega) J(\omega) / \pi$ with the spectral density $J(\omega)$ and the thermal occupation number $n_{\beta}(\omega)$ of the boson bath with temperature $1 / \beta$. In numerical studies of the rate equation (4) one has to use a finite basis of Floquet states. The validity of this approximation is discussed in Ref. [18].

\section{THE SWITCHING MECHANISM}

We will demonstrate the switching process by studying the asymptotic spatial probability density averaged over one period of the driving

$$
\rho(x):=\lim _{t \rightarrow \infty} \frac{1}{T} \int_{t}^{t+T} \mathrm{~d} t^{\prime}\left\langle x\left|\rho\left(t^{\prime}\right)\right| x\right\rangle .
$$

It can be expressed in terms of the solutions $\rho_{i j}$ of Eq. (4) by $\rho(x)=\sum_{i, j} \rho_{i j} \frac{1}{T} \int_{0}^{T} \mathrm{~d} t u_{j}^{*}(x, t) u_{i}(x, t)$. Figure प(b) shows that for the undriven double well, $A=0$, in thermodynamic equilibrium at low temperatures almost all probability is in the left well. This reflects the dominant occupation of the ground state. Figure 1(c) shows that for a small driving amplitude, $A_{0} \approx 0.008$, the probability density is almost completely transferred to the right well. Note, that the driving amplitude is so small, that at all times the right well is energetically higher than the left well. This example demonstrates that a weak periodic driving not only alters the static Boltzmann occupation probabilities [15, 18], but can switch to an almost orthogonal and macroscopically different asymptotic state of the system.

We get a first insight into this dramatic phenomenon from Figs. 2(a) and (b), where one can see that under 
(a)

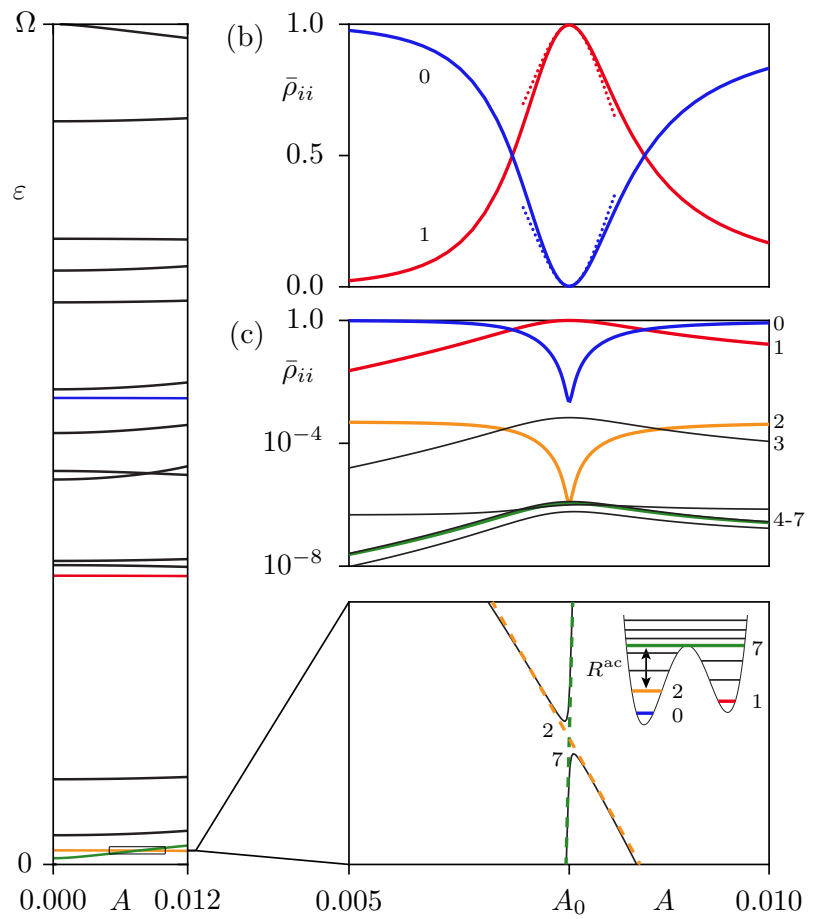

FIG. 2: a) Quasienergy spectrum for the 17 lowest Floquet states vs. driving strength $A$ and magnification of the avoided crossing at $A=A_{0}$ (solid lines) with $\Delta=\left|\varepsilon_{2}\left(A_{0}\right)-\varepsilon_{7}\left(A_{0}\right)\right| \approx$ $1.82 \cdot 10^{-6}$, the quasienergies corresponding to diabatic states 2 and 7 (dashed lines), and eigenenergies of the undriven potential (inset). b) Stationary occupations $\bar{\rho}_{i i}$ in the diabatic basis (solid lines) and approximation based on effective rate $R^{\text {ac }}$, Eqs. (6) and (7) (dotted lines). c) same as b) with logarithmic axis for $\bar{\rho}_{i i}$. The parameters are $\mu=0.03, \hbar_{\text {eff }}=0.04$, $\Omega=\hbar_{\text {eff }} / 0.768, \beta=100, \eta=10^{-4}$ and $\omega_{c}=100$.

the variation of the driving amplitude $A$ the quasienergy spectrum shows around $A=A_{0}$ an isolated avoided crossing of the states 2 and 7 originating from the second and the 7th excited state of the undriven system. We emphasize, that both the ground state, which is dominantly populated at $A=0$, and the first excited state, which will turn out to be dominantly populated at $A=A_{0}$, are not involved in this avoided crossing.

An intuitive understanding of the switching from the rate equation seems impossible: Tuning through an avoided crossing of the two Floquet states 2 and 7 they exchange their character and thus drastically affect in Eq. (4) a large number of rates $R_{i j ; k l}$, where one of the four indices is 2 or 7 . In order to visualize the changes of the density operator due to this avoided crossing it is convenient to express this operator in a basis that does not significantly change in the neighborhood of the avoided crossing. In the subspace of the Floquet states of the avoided crossing we use the diabatic states 2 and 7 , which would correspond to an exact crossing. Due to the weak driving amplitude $A \ll \mu$ they are nearly identical to the eigenstates of the undriven system (Fig. 2(b), inset). We will denote quantities in this diabatic basis by a bar.

The diagonal density matrix elements $\bar{\rho}_{i i}$ in the diabatic basis are shown in Fig. 2(b) and (c). One observes that $\bar{\rho}_{00}$, which corresponds to being in the ground state of the undriven system, drops from close to one to almost zero for $A=A_{0}$. In contrast, the probability $\bar{\rho}_{11}$ increases almost to one, which corresponds to the first excited state being dominantly populated. The tiny occupations $\bar{\rho}_{22}$ and $\bar{\rho}_{77}$, i.e. the probabilities to be in one of the states of the avoided crossing, become equal. These observations for $\bar{\rho}_{i i}$ are consistent with the spatial probability density observed in Fig. 1(c) and can indeed be exploited for a switching between the wells: Tuning the driving amplitude from outside the avoided crossing into its center is accompanied by a probability transfer from the former ground state in the left well to the first excited state localized in the right well.

While the equality $\bar{\rho}_{22} \simeq \bar{\rho}_{77}$ at the center of an avoided crossing of states 2 and 7 is quite plausible, the main question is still unanswered: How can states 0 and 1, which are not involved in the avoided crossing, interchange their probability?

\section{A. Effective rate equations}

We will answer the above question by using an effective approximate rate system introduced in Ref. [18], which is derived from Eq. (4),

$$
0=-\bar{\rho}_{i i} \sum_{k} \bar{R}_{i k}+\sum_{k} \bar{\rho}_{k k} \bar{R}_{k i},
$$

for the diagonal elements $\bar{\rho}_{i i}$ in the diabatic basis with an additional rate

$$
R^{\mathrm{ac}}:=\frac{\Gamma}{(\Gamma / \Delta)^{2}+4 d^{2}}
$$

replacing the rates $\bar{R}_{27}, \bar{R}_{72}$ in Eq. (6) due to the single isolated avoided crossing of diabatic states 2 and 7 . Before we make use of these equations, we make a number of remarks: The rates $\bar{R}_{i k} \equiv \bar{R}_{i k ; i k}$ are expressed in the diabatic basis. The rate $\Gamma=\Gamma_{2}+\Gamma_{7}+\bar{R}_{22}+\bar{R}_{77}-2 \bar{R}_{22 ; 77}$ with $\Gamma_{i}=\sum_{k \neq i} \bar{R}_{i k}(i=2,7)$ describes the transitions from the states of the avoided crossing to all other states. It is proportional to $\eta$ with a factor that is specific to an individual avoided crossing. The rate $R^{\text {ac }}$ depends on the minimal splitting $\Delta$ of the avoided crossing and the dimensionless distance $d:=\left(\bar{\varepsilon}_{7}-\bar{\varepsilon}_{2}\right) / \Delta$ from the avoided crossing. The main assumptions used in the derivation [18] is that all quasienergy splittings $\varepsilon_{i j}$, apart from the isolated avoided crossing of interest, are much larger than the rates $R_{i j ; k l}$. This is fulfilled for a sufficiently weak coupling to the heat bath and allows for neglecting almost all off-diagonal density matrix elements. The only non-negligible off-diagonal elements are $\bar{\rho}_{27}$ and $\bar{\rho}_{72}$, which are decoupled from Eq. (6) and proportional to $\bar{\rho}_{22}-\bar{\rho}_{77}$. The dotted lines in Figs. 2(b) demonstrate this approximation. 
The main advantage of the effective rate system in the diabatic basis, Eq. (6), is, that tuning the distance $d$ from the avoided crossing affects exclusively the rate $R^{\text {ac }}$. In the center of the avoided crossing, $d=0$, and for a small enough coupling to the heat bath, $\Gamma \ll \Delta$, it is much larger than all other rates. This leads directly to almost equal occupations of the diabatic states involved in the avoided crossing, $\bar{\rho}_{22} \simeq \bar{\rho}_{77}$. We explain the dominant occupation of state 1 as the combined result of the following facts: (i) The overall stationary probability flux between any two states is in general nonzero, as detailed balance is broken by the periodic driving. (ii) The rates between neighboring states localized in the same well are much larger than other intra-well rates as well as interwell rates (due to the small spatial overlap between the states of different wells). Therefore, among the states confined to the same well detailed balance approximately holds true, e.g. between state 0 and 2 or state 1 and 3 . (iii) $R^{\text {ac }}$ is the dominant rate and induces occupation equality of states 2 and 7 . Figure 2(c) shows the resulting depopulation of state 2 towards state 7. (iv) Due to the approximate detailed balance among the states in the left well the relative occupation of state 0 and 2 remains constant and therefore $\bar{\rho}_{00}$ drops down together with $\bar{\rho}_{22}$. (v) The states in the right well equilibrate as before but with increased weights due to probability conservation. This explains the switching process observed in Fig. 1.

An additional surprising phenomenon is observed in Fig. 2(b). The impact of the avoided crossing on the occupations occurs within a significantly broader range of the driving amplitude $A$ compared to the width of the avoided crossing. For the parameters of Fig. 2 the full width at half maximum of $P_{r}(A)=\int_{0}^{\infty} \mathrm{d} x \rho(x)$, the probability to be in the right potential well (Fig. 3(a)), is a factor of 30 larger than the width of the avoided crossing. According to Eqs. (6) and (77), the occupations change, if the magnitude of $R^{\text {ac }}$ is larger than or comparable to other significant rates in Eq. (6). Since these rates vary over many orders of magnitude, this criterion may be fulfilled even beyond the avoided crossing, $|d|>1$, qualitatively explaining the enlarged width of $P_{r}(A)$.

\section{B. Parameter dependence}

What are the optimal parameters for this switching effect? A maximal switching efficiency is achieved by a high value of the probability in the right well $P_{r}(A)=$ $\int_{0}^{\infty} \mathrm{d} x \rho(x)$. This quantity is shown in Fig. 3 (a). Figure 3 (b) demonstrates that if the coupling to the heat bath is larger than the minimal splitting of the avoided crossing, $\Gamma>100 \Delta$, almost no probability is switched to the right well. (Note, that even for the largest values of $\Gamma$ in Fig. 3(b) the assumption of weak coupling of the Floquet-Markov approach is still fulfilled.) This is due to the fact that in this limit $R^{\text {ac }}$ becomes negligible compared to the other rates and thus the influence of the avoided crossing vanishes [18]. In contrast, for small cou-
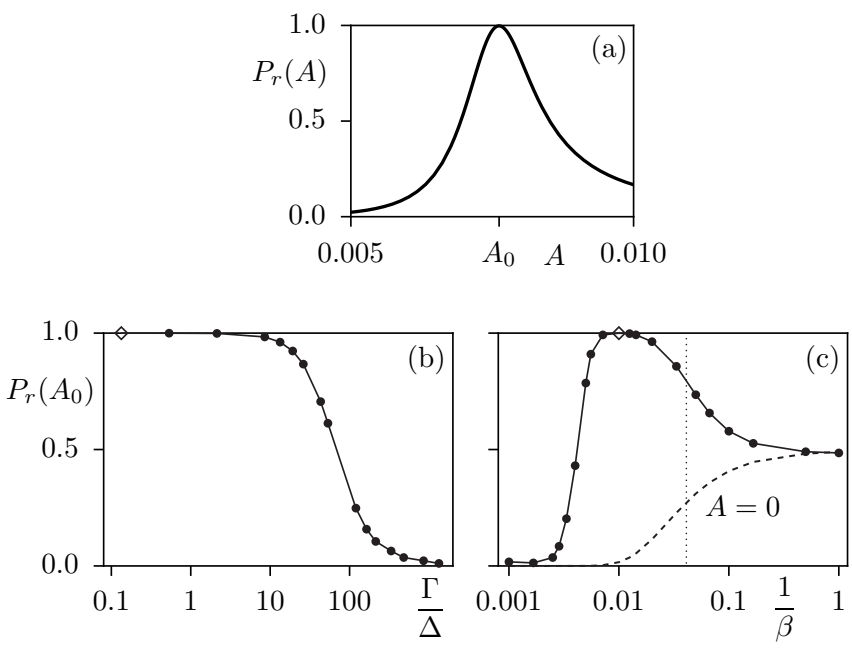

FIG. 3: a) Total probability in the right well $P_{r}$ vs. driving amplitude $A$. b), c) Peak height $P_{r}\left(A_{0}\right)$ vs. effective coupling strength $\Gamma / \Delta$ and temperature $1 / \beta$. Diamonds indicate the parameters of Fig. 2 The dashed line in c) gives the probability in the right well without driving, $A=0$. The dotted line at $1 / \beta=E_{1}-E_{0}$ indicates the transition between the high and the low-temperature regimes.

pling $\Gamma<\Delta$ we have a high switching efficiency and one can show that it is independent of $\Gamma$ in the limit $\Gamma \rightarrow 0$.

Figure 3(c) shows the influence of the temperature $1 / \beta$, which can be related to the level spacing $E_{1}-E_{0}$ of the undriven system. At high temperatures, $1 / \beta \gg E_{1}-E_{0}$, the Floquet states are almost equally occupied resulting in $P_{r}\left(A_{0}\right) \approx 0.5$. For temperatures $1 / \beta<E_{1}-E_{0}$ the probability in the right well becomes dominant (while, of course, it vanishes in the undriven case $A=0$ ). For even lower temperatures, however, $P_{r}\left(A_{0}\right)$ drops to zero. Here, the occupation equality $\bar{\rho}_{22} \simeq \bar{\rho}_{77}$ is rendered by an increase of $\bar{\rho}_{77}$ towards $\bar{\rho}_{22}$, in contrast to the decrease of $\bar{\rho}_{22}$ towards $\bar{\rho}_{77}$ in Fig. 2(c). Together with $\bar{\rho}_{22}$ also $\bar{\rho}_{00}$ remains constant and therefore switching does not take place. The origin of this low-temperature dependence remains open.

\section{Minimal example}

A minimal example, where one of the partners of the avoided crossing is the ground state in the left well, is shown in Fig. 4. The above discussion then simplifies since step (iv) is eliminated and essentially just three states are involved. The parameter dependence of $P_{r}\left(A_{0}\right)$ on the effective coupling strength $\Gamma / \Delta$ is unchanged and the switching mechanism is maintained even for low temperatures, see Fig. 5 .

For a clear presentation we have above chosen examples in the limit of a small driving amplitude, where the Floquet states are not very different from the eigenstates of the undriven system. In this case an avoided crossing requires near-resonant driving, $E_{7}-E_{2} \approx 3 \Omega$ in Fig. 2 
(a)

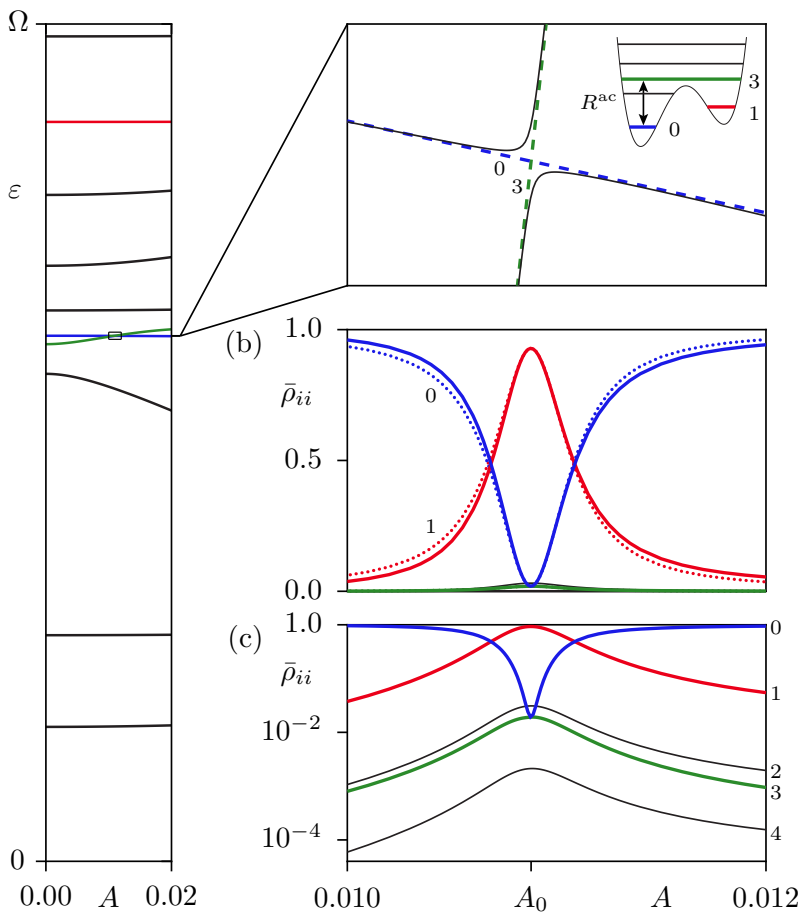

FIG. 4: a) Quasienergy spectrum for the ten lowest Floquet states vs. driving strength $A$ and magnification of the avoided crossing at $A=A_{0}$ (solid lines) with $\Delta=\left|\varepsilon_{0}\left(A_{0}\right)-\varepsilon_{3}\left(A_{0}\right)\right| \approx$ $3.57 \cdot 10^{-6}$, the quasienergies corresponding to diabatic states 0 and 3 (dashed lines), and eigenenergies of the undriven potential (inset). b) Stationary occupations $\bar{\rho}_{i i}$ in the diabatic basis (solid lines) and approximation based on effective rate $R^{\text {ac }}$, Eqs. (6) and (7) (dotted lines). c) same as b) with logarithmic axis for $\bar{\rho}_{i i}$. The parameters are $\mu=0.08, \hbar_{\text {eff }}=0.1$, $\Omega=0.08165, \beta=60, \eta=10^{-6}$ and $\omega_{c}=100$.
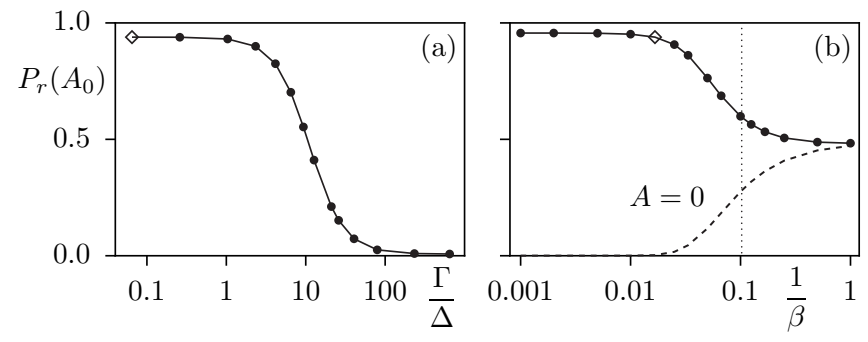

FIG. 5: a), b) Peak height $P_{r}\left(A_{0}\right)$ of the total probability in the right well vs. effective coupling strength $\Gamma / \Delta$ and temperature $1 / \beta$. Diamonds indicate the parameters of Fig. 4. The dashed line in $\mathrm{b}$ ) gives the probability in the right well without driving, $A=0$. The dotted line at $1 / \beta=E_{1}-E_{0}$ indicates the transition between the high and the low-temperature regimes. and $E_{3}-E_{0} \approx 3 \Omega$ in Fig. 4 We have observed switching also in the case of strong driving, supporting the generality of the proposed switching mechanism.

\section{CONCLUSION}

In conclusion, we demonstrate a new switching mechanism for an asymmetric double well potential under a weak periodic driving and a weak coupling to a heat bath. As the origin of the switching we identify an avoided crossing in the quasienergy spectrum of the system. Under its influence the asymptotic occupations of all Floquet states dramatically change even if both involved Floquet states have just small occupations. We explain this switching mechanism by an effective rate equation at the avoided crossing.

We now briefly discuss possible advantages of the switching mechanism in applications: (i) If one uses a laser for the periodic driving, the amplitude dependence of the switching mechanism and the beam profile allow switching at a 3D spatially localized position with a resolution smaller than the focus width. (ii) In situations where a theoretical modeling of the system, e.g. a complex molecule, is not achievable and no other switching mechanism is known, the generic appearance of avoided crossings in time-periodically driven systems suggests the existence of driving parameters for the desired switching.

We emphasize that this switching mechanism is completely different from standard techniques which allow to transfer a wave packet from one well to the other by resonant or near resonant driving and negligible coupling to a heat bath. There one has to prepare a specific initial wave packet and has to apply the driving for a specific duration. In contrast, here the initial state of the system is arbitrary, the duration of the driving is arbitrary (if larger than the relaxation time), and the presence of the heat bath is essential.

\section{Acknowledgements}

We acknowledge helpful discussions with D. Hone, S. Kohler, and W. Kohn. R.K. thanks the Kavli Institute for Theoretical Physics at UCSB (NSF Grant No. PHY05-51164).
[1] M. Grifoni and P. Hänggi, Phys. Rep. 304, 229-354 (1998).

[2] S. Kohler, R. Utermann, P. Hänggi, and T. Dittrich, Phys. Rev. E 58, 7219-7230 (1998).

[3] D. E. Makarov and N. Makri, Phys. Rev. E 52, 58635872 (1995).
[4] D. Solenov and V. A. Burdov, Phys. Rev. B 72, 085347 (2005).

[5] Y. Makhlin, G. Schön, and A. Shnirman, Rev. Mod. Phys. 73, 357-400 (2001).

[6] E. Kierig, U. Schnorrberger, A. Schietinger, J. Tomkovic, and M. K. Oberthaler, Phys. Rev. Lett. 100, 190405 
(2008).

[7] I. H. Deutsch, P. M. Alsing, J. Grondalski, S. Ghose, D. L. Haycock, and P. S. Jessen, J. Opt. B: Quantum Semiclass. Opt. 2, 633-644 (2000).

[8] R. Lü and J. von Delft, Phys. Rev. B 67, 104425 (2003).

[9] A. Douhal, F. Lahmani, and A. H. Zewail, Chem. Phys. 207, 477-498 (1996).

[10] H. Adam, M. Winterstetter, M. Grifoni, and U. Weiss, Phys. Rev. Lett. 83, 252 (1999).

[11] M. C. Goorden, M. Thorwart, and M. Grifoni, Phys. Rev. Lett. 93, 267005 (2004).

[12] I. Goychuk and P. Hänggi, Adv. in Physics 54, 525-584 (2005).

[13] T. M. Stace, A. C. Doherty, and S. D. Barrett, Phys. Rev. Lett. 95, 106801 (2005).

[14] R. Blümel, A. Buchleitner, R. Graham, L. Sirko, U. Smilansky, H. Walther, Phys. Rev. A 44, 4521-4540 (1991).
[15] S. Kohler, T. Dittrich, and P. Hänggi, Phys. Rev. E 55, 300-313 (1997).

[16] H.-P. Breuer, W. Huber, and F. Petruccione, Phys. Rev. E 61, 4883-4889 (2000).

[17] W. Kohn, J. Stat. Phys. 103, 417-423 (2001).

[18] D. W. Hone, R. Ketzmerick, and W. Kohn, Phys. Rev. E 79, 051129 (2009).

[19] M. Thorwart and P. Jung, Phys. Rev. Lett. 78, 2503 (1997).

[20] M. Thorwart, P. Reimann, P. Jung, and R. F. Fox, Phys. Lett. A 239, 233-238 (1998); Chem. Phys. 235, 61-80 (1998).

[21] U. Weiss: Quantum Dissipative Systems, Vol. 10 of Series in Modern Condensed Matter Physics (World Scientific, Singapore, 1999). 